\title{
First order magneto-structural transition in functional magnetic materials: phase-coexistence and metastability
}

\author{
S B ROY*, M K CHATTOPADHYAY, M A MANEKAR, K J S SOKHEY and \\ P CHADDAH ${ }^{\dagger}$ \\ Magnetic and Superconducting Materials Section, Raja Ramanna Centre for Advanced Technology, \\ Indore 452 013, India \\ ${ }^{\dagger}$ UGC-DAE Consortium for Scientific Research, University Campus, Khandwa Road, Indore 452 017, India
}

\begin{abstract}
First order magneto-structural transition plays an important role in the functionality of various magnetic materials of current interest like manganese oxide systems showing colossal magnetoresistance, $\mathrm{Gd}_{5}(\mathrm{Ge}, \mathrm{Si})_{4}$ alloys showing giant magnetocaloric effects and magnetic shape memory alloys. The key features of this magneto-structural transition are phase-coexistence and metastability. This generality is highlighted with experimental results obtained in a particular class of materials. A generalized framework of disorder influenced first order phase transition is introduced to understand the interesting experimental results which have some bearing on the functionality of the concerned materials.
\end{abstract}

Keywords. Magneto-structural transition; phase-coexistence; metastability.

\section{Introduction}

Magnetic materials are used widely in electric motors, loudspeakers, transformers, automobiles, magnetic resonance devices, magnetic memory storage and a diverse range of scientific instruments from large particle-accelerators to tiny multimeters. The continuous evolution in the field of magnetic materials which are important (but somewhat hidden) component of modern technology goes almost unnoticed. Often classes of magnetic materials are discovered with new interesting functionality, which stimulates the growth of newer technology. The recent research activities pertaining to certain classes of magnetic materials narrated below provide a platform to discuss such emerging science and technology involving newer classes of functional magnetic materials.

\section{Magnetic materials with first order phase transition (FOPT)-driven functionality}

Three classes of magnetic materials have emerged during last two decades with much promise for immediate technological applications. These are: (I) giant magnetoresistance and colossal magnetoresistance materials, (II) magnetocaloric materials and (III) magnetic shape memory alloys. A brief description of these functional materials is given below.

(I) Magnetoresistance, the change in electrical resistance with an applied magnetic field, is a useful tool in several

\footnotetext{
*Author for correspondence (sbroy@ cat.ernet.in)
}

areas of technology. For example, the computer hard drives use magnetoresistance to read the stored data. Most laptop computers now come fitted with high capacity hard drives which use giant magnetoresistance (GMR) sensors as read head. The basic GMR device consists of a three-layer sandwich of a magnetic metal such as cobalt or iron with a nonmagnetic metal filling such as silver or platinum (Prinz 1998). It is interesting to note that the research in such artificially engineered magnetic multi-layers actually started in order to shed light on the fundamental questionhow do magnetic moments interact in magnetic materials? This is a reasonably difficult question in natural solids, which is still fascinating the researchers. The discovery of interesting GMR properties in these artificially engineered materials was rather unanticipated. It came as a bonus out of such curiosity driven basic research and the unforeseen technological development from then on was quite fast. From the first report of the GMR properties in 1988 it took less than ten years for the first product in the form of 'read heads' for computer hard disk drives to have major economic impact. This interesting development naturally spurred more research activity in magnetoresistance materials, and in early 1990s a class of rare-earth manganese oxide materials (commonly termed as manganites) were found with colossal magnetoresistance (CMR) properties (Dagotto 2002). Manganites show exotic physical properties in the form of metal-insulator transition and varieties of magnetic, charge and orbital orderings dictated by strong electron-electron interaction and electron-lattice interaction, and provide a challenging area of research. While the phenomenology of the CMR effect in manganites can be explained within the framework of microscopic double 
exchange interaction, this mechanism alone is insufficient to explain the observed effects quantitatively (Dagotto 2002). A prospective picture in this regard is the formation of a percolation path involving the metallic ferromagnetic (FM) and insulating antiferromagnetic (AFM) phases across a FM-AFM transition region which can be manipulated by an applied magnetic field. This magnetic transition region in manganites has been a subject of much scrutiny in recent times using various experimental techniques including microscopic imaging with electron and magnetic-force microscopy. Distinct phase-coexistence in micrometer scale has been reported leading to the actual visualization of a percolating path (Dagotto 2002). In addition, the lattice distortions and long-range strains are known to be important for manganites and the intrinsic complexity of a system with strong coupling between electronic and elastic degrees of freedom introduces further interesting feature in the phase-coexistence (Ahn et al 2004).

(II) Originally measured in iron, the magnetic field induced temperature variation in a magnetic solid is known as 'magnetocaloric effect' (MCE). Instead of a working fluid undergoing a liquid-vapour transition in conventional refrigerator, a magnetic refrigerator can be envisioned using a magnetic solid which heats up when magnetized and cools down when demagnetized. Such magnetic cooling has a potential to reduce global energy consumption and minimize the need of ozone depleting and greenhouse chemicals. In a suitable MCE material, randomly oriented magnetic moments are aligned by the application of an external magnetic field, resulting in the reduction of magnetic entropy. In turn, the material is heated via increase of its lattice entropy. This heat is removed from the material to its surroundings by a heat-transfer medium. On removing the magnetic field, the magnetic moments become randomized causing an increase in the magnetic entropy. This leads to cooling of the MCE material below the ambient temperature. Using a heat-transfer medium, heat from the system to be cooled can then be extracted. The prospect of magnetic cooling as a viable alternative to vapour-compression technology has increased enormously since the recent discovery of giant MCE in various classes of magnetic materials (Pecharsky and Gschneidner 2001). The origin of this giant MCE is now traced to an interesting magneto-structural transition (Pecharsky et al 2003).

(III) Shape memory alloys (SMA) are metals that have the ability to remember a predetermined shape, and to return back to that shape after being bent, stretched or otherwise mechanically deformed (Bhattacharya 2003). This shape-memory effect is caused by a 'thermoelastic martensitic transition'-a reversible transition between two different crystal microstructures in the concerned metallic system. SMAs have a wide range of technological applications including aeronautical, robotics and biomedical implants. Although the first recorded observation of the shape memory effect dates back to early 1930s, it was not until 1962, when this effect was discovered in equiatomic nickel-titanium (NiTi) alloys, that research into both the science and potential practical uses began in earnest. Within 10 years, a number of commercial products were on the market, and understanding of the effect was much advanced. Study of SMAs has continued at an increasing pace since then, and more products using these materials are coming to the market each year. One of the drawbacks of conventional SMAs is that they are slower to respond because they rely on variations in temperature and the flow of heat. A class of materials has now been discovered in late 1990s which can undergo large reversible deformations in an applied magnetic field. These materials are now known as magnetic shape memory alloys (MSMA) (Ullakko et al 1996; O’Handley 1998). Compared to the ordinary SMAs the magnetic control offers faster response in the MSMAs.

Taken together, many common experimental features from these quite distinct types of materials systems, CMR manganites, giant MCE materials and MSMAs, are indications of a common underlying physics at least at the phenomenological level. The present authors believe that the disorder-influenced first order phase transition (FOPT) provides the basic framework to understand the wide varieties of experimental results in these different classes of functional magnetic materials. This idea of generality is mainly developed on the basis of experimental work performed by these researchers on another class of magnetic materials viz. doped-CeFe $\mathrm{C}_{2}$ alloys. These relatively simple doped $\mathrm{CeFe}_{2}$ alloys (Roy and Coles 1989, 1990) with magneto-structural transition (Kennedy and Coles 1990) have been used as test-bed materials systems to study in some detail, a first order ferromagnetic (FM) to antiferromagnetic (AFM) transition. Based on detailed study of a.c. susceptibility, d.c. magnetization and magnetotransport, it is shown that the key features associated with the AFM-FM transition in manganites and MCE materials are clearly observed in this system and that these are a consequence of phase-coexistence and metastability arising out of a disorder-influenced FOPT. A brief summary of this work is narrated below. To extend the same idea to MCE and MSMA, the researchers have studied the magneto-martensitic transition in prototype MCE material, $\mathrm{Gd}_{5} \mathrm{Ge}_{4}$ (Chattopadhyay et al 2004; Moore et al 2006) and new ternary alloy systems, NiCoAl (Sokhey et al 2003) and NiFeGa (Majumdar et al 2005), showing magnetic shape memory effect. However, details of these studies will not be discussed here.

\section{Doped-CeFe $\mathrm{C}_{2}$ alloys - a test bed material system to study disorder influenced FOPT}

Figure 1 shows the a.c. susceptibility $(\chi)$ for $\mathrm{Ir}$ and $\mathrm{Ru}-$ doped $\mathrm{CeFe}_{2}$ samples as a function of temperature $(T)$ 

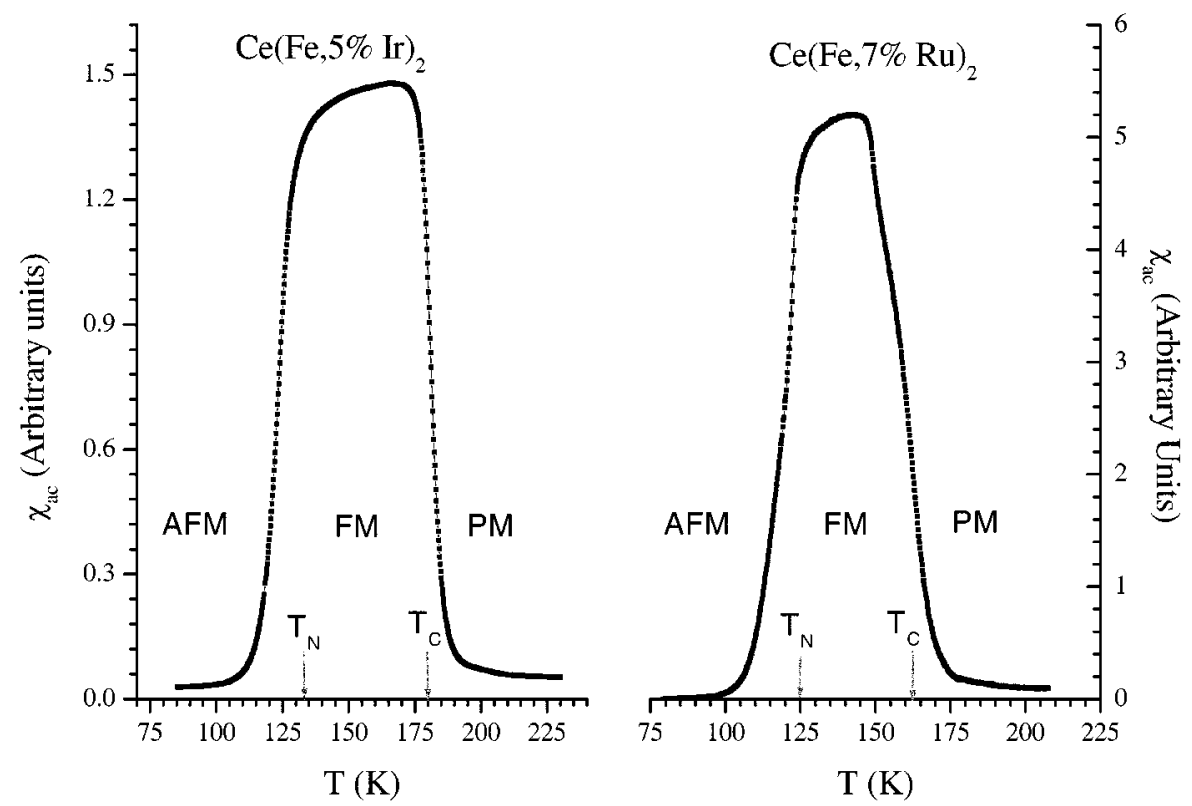

Figure 1. AC susceptibility $(\chi)$ vs temperature $(T)$ plots for Ir and $\mathrm{Ru}$-doped $\mathrm{CeFe}_{2}$ alloys (Manekar et al 2000a).

(Manekar et al 2000a). A sharp increase in $\chi$ at a particular temperature, $T_{\text {Curie }}$, while decreasing $T$ marks the onset of paramagnetic (PM)-FM transition. Below $T_{\text {Curie, }}$, susceptibility more or less flattens out before decreasing sharply at a lower temperature, $T_{\mathrm{N}}$, which is indicative of an FM-AFM transition. There is no effect of thermal cycling on the PM-FM transition and this is in accord with the second order nature of this transition. Within the same experimental resolution, however, distinct thermal hysteresis is observed across the FM-AFM transition (Manekar et al 2000a). This is necessarily a signature of FOPT. The phase coexistence across this FOPT is studied in detail using minor hysteresis loop (MHL) technique (Manekar et al 2000a, b). This technique was developed earlier in the context of research in vortex matter phase transition in type-II superconductors (Roy and Chaddah 1997a, b).

Figure 2 shows magnetization $(M)$ vs temperature $(T)$ plot for a $4 \% \mathrm{Ru}$-doped $\mathrm{CeFe}_{2}$ sample in an applied field of $20 \mathrm{kOe}$ (Sokhey et al 2004). Three different measurement protocols were used: zero-field cooled (ZFC), field-cooled cooling (FCC) and field-cooled warming (FCW). A rapid rise of $M$ with decreasing $T$ below $\sim 210 \mathrm{~K}$ indicates the onset of PM-FM transition and it is thermally reversible. The FM-AFM transition is marked by the sharp drop in $M$ below $50 \mathrm{~K}$ and shows substantial thermal hysteresis, which is necessarily a signature of FOPT. It should be noted that the FCC curve does not merge with the ZFC curve down to the lowest measured temperature of $5 \mathrm{~K}$. Similar measurements with applied fields varying between $100 \mathrm{Oe}$ and $30 \mathrm{kOe}$ shows that thermal hysteresis broadens with increasing $H$, and when $H \geq 15 \mathrm{kOe}$ the
$M_{\mathrm{FCC}}(T)$ and $M_{\mathrm{ZFC}}(T)$ curves fail to merge. The onset of the FM-AFM transition gives rise to marked increase in electrical resistivity (see inset of figure 3). The FM-AFM transition can be suppressed by an applied $H$ and this gives rise to GMR effect which is clearly visible in figure 3 . The schematic $H-T$ phase diagram based on the magnetization measurements for this $4 \% \mathrm{Ru}$-doped $\mathrm{CeFe}_{2}$ alloy is shown in figure 4 with $T_{\mathrm{NW}}\left(T_{\mathrm{NC}}\right)$ as the temperature of the sharp rise (fall) in $M$ in the ZFC (FCC) cycle (see inset of figure 4). $T^{*}$ is the low $T$ point where $M_{\mathrm{ZFC}}$ and $M_{\mathrm{FC}}$ merges and $T^{* *}$ is the high $T$ counterpart. $T^{* *}$ and $T_{\mathrm{NC}}$ appear almost the same in our present magnetization measurements. Similar $H-T$ phase diagram can be obtained through resistivity measurements and in such measurements (under same experimental protocol), $T^{* *}$ and $T_{\mathrm{NC}}$ can be distinguished clearly (Sokhey et al 2004). Note that $T_{\mathrm{NW}}(H)<T_{\mathrm{NC}}(H)$, i.e. the onset of nucleation of the AFM state on cooling occurs at a higher temperature than does nucleation of the FM phase during warming. This is a signature of a disorder-broadened FOPT. Such influence of disorder is observed in the same sample in the field induced transition also and this is discussed in detail in an earlier work (Chattopadhyay et al 2003). Figure 5 shows schematic curves of the free energy density expressed in terms of an order parameter, $S$, as $f(T, S)=(r / 2) S^{2}-$ $w S^{3}+u S^{4}$ for a first order transition, where $w$ and $u$ are positive temperature independent constants. At $T=T_{\mathrm{N}}$, the high- $T$ and low- $T$ phases coexist. The standard treatment (Chaikin and Lubensky 1995; Chaddah and Roy 1999) assumes that $r(T)=a\left[T-T^{*}\right]$, where $a$ is positive and temperature independent, where $d^{2} f / \mathrm{d} S^{2}$ at $S=0$ vanishes at $T=T^{*}$. The limit of metastability on cooling is 


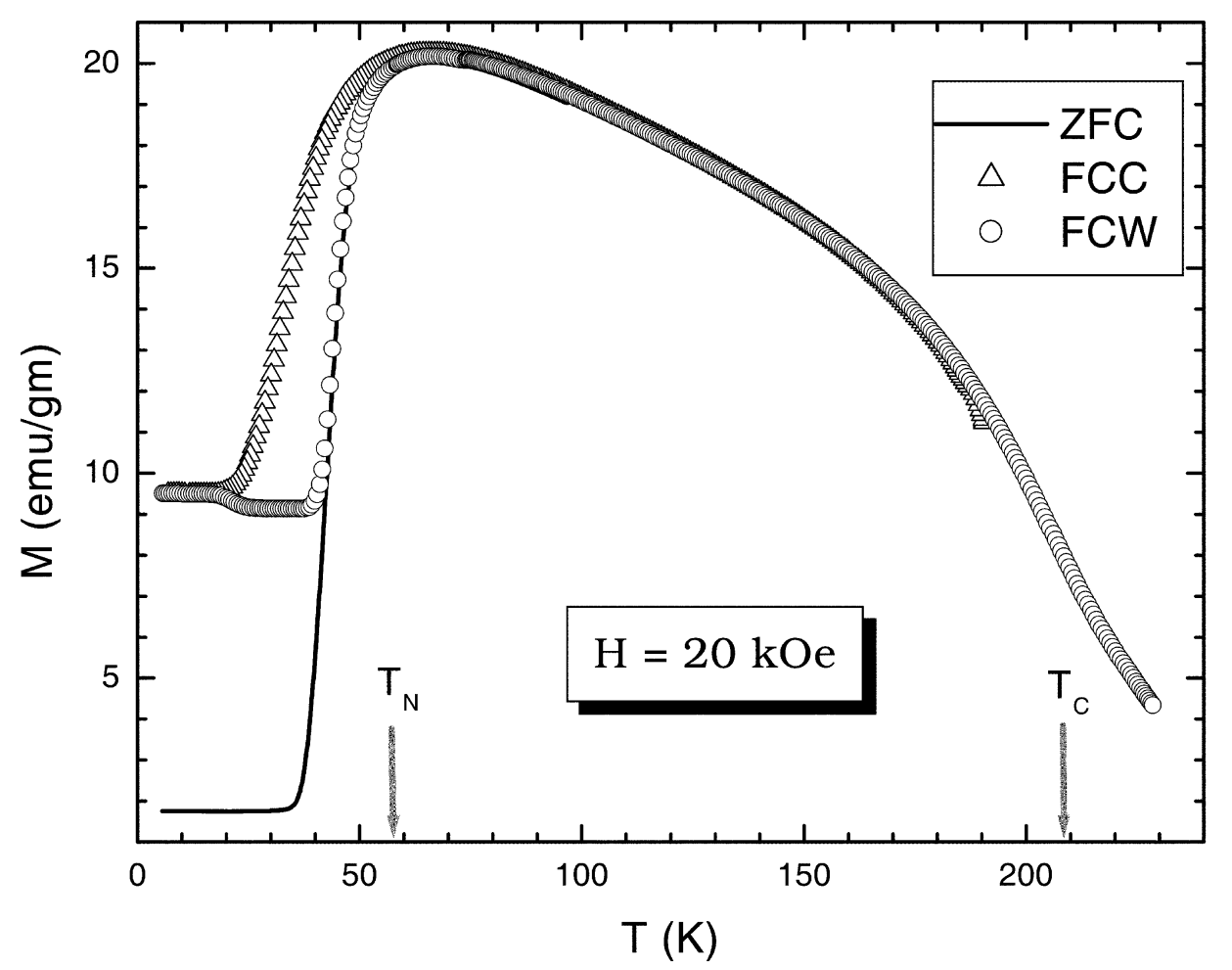

Figure 2. $\quad M$ vs $T$ plots for $\mathrm{Ce}\left(\mathrm{Fe}_{0 \cdot 96} \mathrm{Ru}_{0 \cdot 04}\right)_{2}$ alloy (Sokhey et al 2004).

reached at $T^{*}=T_{\mathrm{N}}-w 2 /(2 u a)$, but finite energy fluctuations can destroy the supercooled state in the temperature regime $T^{*}<T<T_{\mathrm{N}}$. Similarly $T^{* *}$ is the limit of metastability while warming. In the low temperature AFM regime a transition from the zero field AFM state to FM state can be induced by application of $H$ (Chattopadhyay et al 2003; Roy et al 2005). As in the case of temperature variation, this field induced first order AFM-FM transition is also marked by distinct hysteresis and phase-coexistence and accordingly the limits of metastability, $H^{*}$ and $H^{* *}$, can be defined.

$H-T$ phase diagram in figure 4 shows that it is possible to retain residual FM state in this $4 \% \mathrm{Ru}$-doped $\mathrm{CeFe}_{2}$ alloy down to the lowest $T$ of measurements by following the FCC path with applied $H \geq 15 \mathrm{kOe}$. This is exactly what is known as field annealing of FM state in manganite systems showing CMR effect (Kimura et al 1999). It has been shown that this residual FM obtained by the FCC path is very much metastable in nature and can be erased by field cycling (Sokhey et al 2004). All these observations can be rationalized in terms of supercooling of the FM state. While cooling across the first order FM-AFM transition, some amount of FM state will supercool into the $T$ regime well below the transition line. It is clear from figure 4 that with applied $H<15 \mathrm{kOe}$ the supercooled FM state will cease to exist below a finite $T$ and one can reach the stable AFM state. This is indicated by the merger of the FC and ZFC magnetization. With $H \geq 15$ kOe some amount of supercooled FM state remains down to the lowest $T$ of measurement. The region between $T_{\mathrm{NC}}(H)$ and $T^{*}(H)$ line in figure 4 marks the phase-coexistence region formed during the cooling path. This region consists of mixtures of AFM and FM clusters and it is metastable in nature. A new concept viz. 'lack of end point memory effect' (Roy et al 2002) has been used to study, in detail, the metastable nature of this phase-coexistence regime (Chattopadhyay et al 2003; Sokhey et al 2004). Any field cycling in this metastable phase-coexistence region (obtained via FCC path) introduces energy fluctuations, which drive the clusters of metastable FM state to the stable AFM state. Further support of metastable nature of the phase-coexistence state have come from large relaxation of both magnetization and resistivity in the phase-coexistence regime (Chattopadhyay et al 2003).

The actual composition in any alloy or doped compound vary around some average composition simply due to the disorder that is frozen in as the solid crystallizes from the melt. It was proposed earlier (Imry and Wortis 1979) that such static, quenched in, purely statistical compositional disorder can under certain circumstances introduce a landscape of transition temperatures in a system undergoing FOPT. Effect of quenched disorder in a FOPT was probably known to the metallurgists for ages. However, a systematic study of the effect of disorder on a FOPT process started only in late 1970s (Imry and Wortis 1979). Detailed computational studies (Dagotto et al 2002) con- 


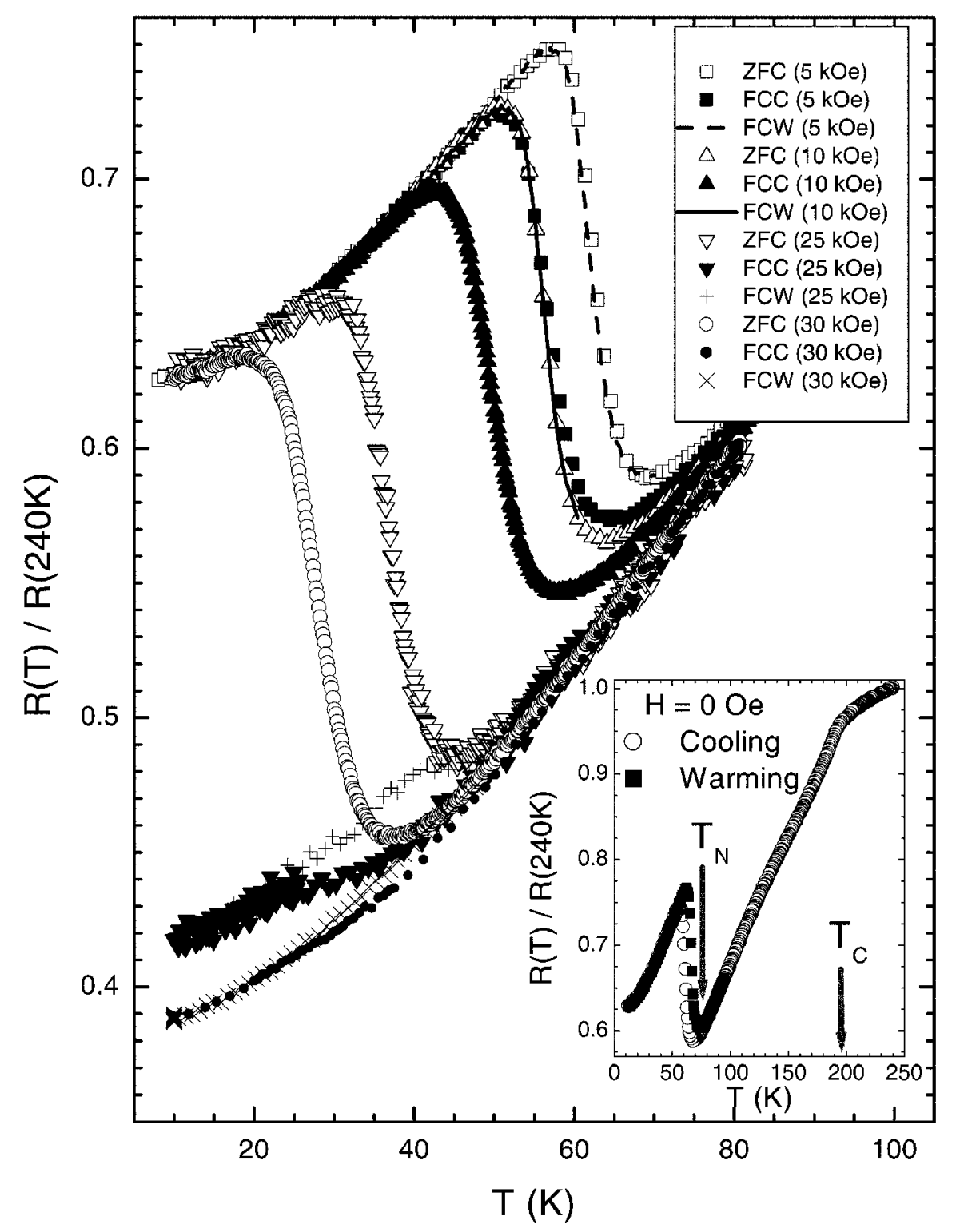

Figure 3. Resistivity ratio vs $T$ plots for $\mathrm{Ce}\left(\mathrm{Fe}_{0.96} \mathrm{Ru}_{0.04}\right)_{2}$ alloy (Sokhey et al 2004).

firm the applicability of disorder-influenced FOPT in CMR manganites and further emphasize that phasecoexistence can occur in any system in the presence of quenched disorder whenever two states are in competition through a FOPT (Dagotto et al 2002). Such intrinsic disorder induced landscape of transition temperature/field has actually been observed across the vortex solid melting transition in a high temperature superconductor, BSCCO (Soibel et al 2000). The applicability of such a picture in the AFM-FM transition of the doped-CeFe 2 alloys has now been pointed out through an imaging study of AFMFM transition using a micro Hall probe (Roy et al 2004). It was observed both in $T$ and $H$ variation measurements in doped-CeFe $\mathrm{Ce}_{2}$ alloys that the FM clusters of various size appear in random positions of the sample at the onset of the AFM-FM transition. As the temperature or field is increased, newer FM clusters appear until the whole sample is converted to the FM state. This is clearly indicative of the local variation of the AFM-FM transition temperature $\left(T_{\mathrm{N}}\right)$ or field $\left(H_{\mathrm{M}}\right)$ leading to a rough $T_{\mathrm{N}} / H_{\mathrm{M}}$ landscape. This distribution of $T_{\mathrm{N}}$ or $H_{\mathrm{M}}$ gives rise to the impression of a global rounding of the transition in bulk measurements. This imaging study also provided a visual proof of supercooling of the FM state across the AFM-FM transition and that such supercooled state can easily be destabilized with a small energy fluctuation (Roy et al 2004). In the less disordered samples the growth process of the clusters is relatively fast with smaller number of nucleating clusters, which suggests that different disorder landscapes can control nucleation and growth with the key point that if 


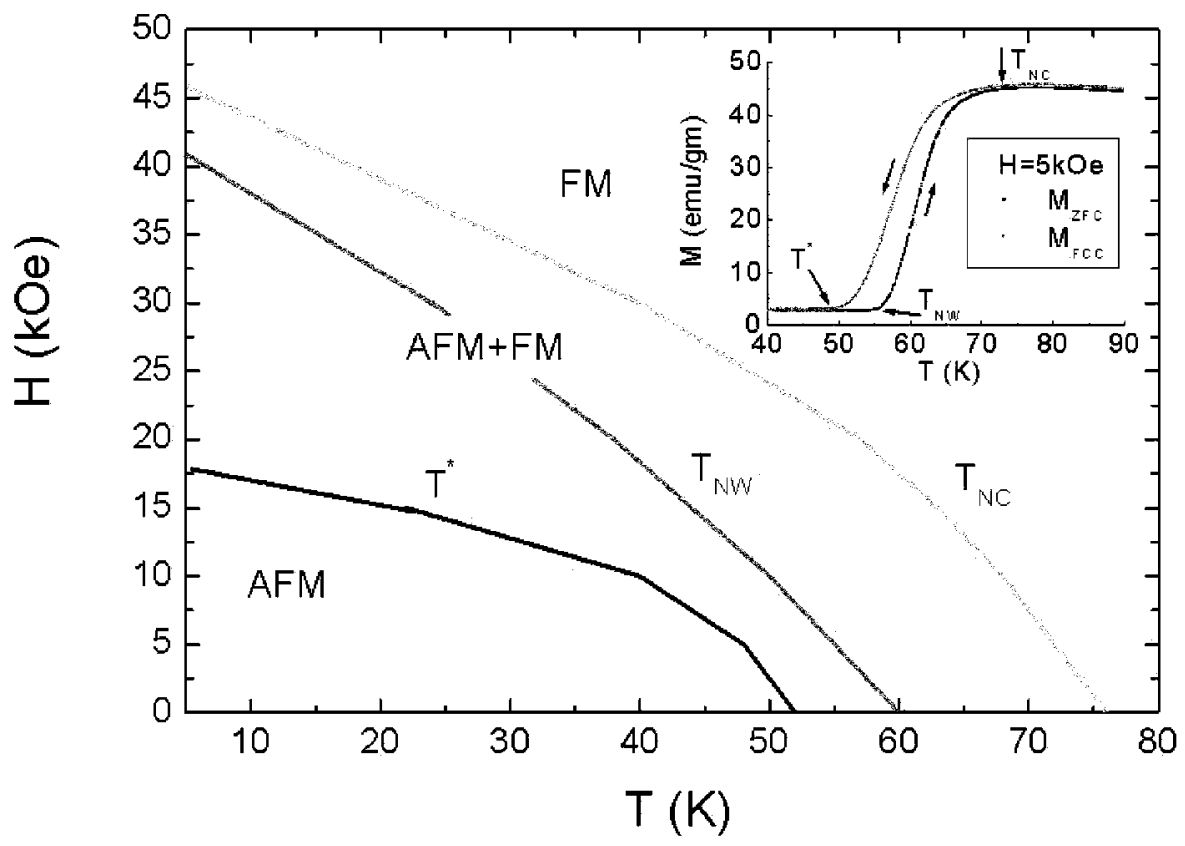

Figure 4. $H-T$ phase diagram for $\mathrm{Ce}\left(\mathrm{Fe}_{0.96} \mathrm{Ru}_{0.04}\right)_{2}$ alloy.

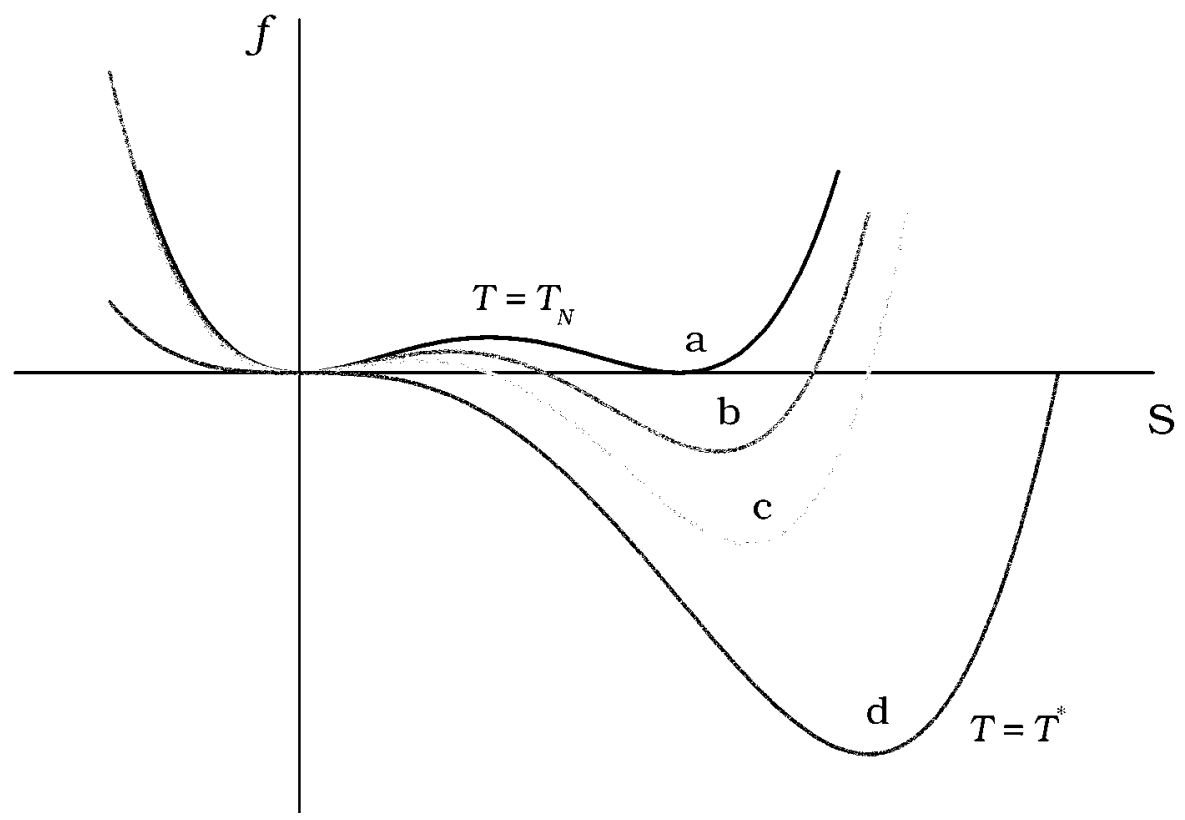

Figure 5. Schematic free energy curves for $T^{*}<T<T_{\mathrm{N}}$. The high temperature state remains in a local minimum and is stable against infinitesimal energy fluctuations in this temperature regime. The barrier height between the minima corresponding to the high and low temperature phases at TN (curve a) decreases with the decrease in $T$ and goes to zero at $T^{*}$ (curve d).

the on-line growth is slow enough, percolation will occur over an observable $T$ or $H$ interval before phase-coexistence collapses. Such percolative behaviour can be controlled by subtle changes in sample doping, and the ramification to tuning the functionality of the CMR-manganite systems, for example, is obvious (Roy et al 2004).
In a nutshell, a first order magneto-structural transition is the common feature in CMR-manganites (Dagotto 2002) systems with giant MCE (Pecharsky and Gschneidner 2001) and ferromagnetic shape memory alloys (Sokhey et al 2003; Majumdar et al 2005). Phase-coexistence and metastability are the essential features of this transition 
process and influences the functionality of these materials. Understanding these phenomena in detail will help in tuning the functionality of the existing materials as well as finding new materials with better functionality.

\section{Kinetic arrest of a FOPT in magnetic systems and a new type of magnetic glass}

Liquids freeze into crystalline solids via a first order phase transition. However, some liquids called 'glass formers' experience a viscous retardation of nucleation and crystallization in their supercooled state. In the experimental time scale the supercooled liquid ceases to be ergodic and it enters a glassy state. In a very similar manner it was seen that the first order FM to AFM transition process could be arrested in a $\mathrm{Ru}$-doped $\mathrm{CeFe}_{2}$ alloy when the applied magnetic field was beyond a certain critical value, and the lowest temperature magnetic state became nonergodic in nature. It should be noted here that the behaviour typical of glass formation is not necessarily restricted to materials that are positionally disordered (Brawer 1984). Even for the conventional glasses, other than the general definition that 'glass is a noncrystalline solid material which yields broad nearly featureless diffraction pattern', there exists another widely acceptable picture of glass as a liquid where the atomic or molecular motions are arrested. Within this latter dynamical framework 'glass is time held still' (Brawer 1984).

To elucidate on the novel magnetic-glass behaviour, some results of magnetization relaxation on various points on the FCC leg of the $M-T$ curve of a $4 \% \mathrm{Ru}$-doped $\mathrm{CeFe}_{2}$ alloy (see figure 2) are discussed here. It was already observed that in the FCC path the FM state persists at temperatures well below $T_{\mathrm{N}}$ as supercooled state (Sokhey et al 2004; Roy et al 2004). This FM state is highly metastable and any energy fluctuations tend to convert it into equilibrium AFM state. As the system goes below $T_{\mathrm{N}}$ and approaches the limit of supercooling $T^{*}$, the barrier height between the metastable (FM) and the equilibrium (AFM) state in the free energy curve decreases and hence a decrease of $M$ with higher relaxation rate is expected. This is actually observed in the $T$-regime, $25-40 \mathrm{~K}$ (Chattopadhyay et al 2005). However, some marked change in relaxation takes place in the $T$-regime below roughly $23 \mathrm{~K}$ (see figure 6). The relaxation rate decreases drastically and below $15 \mathrm{~K}$ the relaxation of $M$ is very small even though the metastable FM state persists. Relaxation data below $23 \mathrm{~K}$ can be fitted well with Kohlrausch-WilliamsWatt stretched exponential function $\left(\varphi \propto \exp \left[-(t / \tau)^{\beta}\right]\right)$ (see figure 6 ), where $\tau$ is characteristic relaxation time and $\beta$ is a shape parameter between $0 \cdot 6$ and $0 \cdot 9$. The characteristic relaxation time, $\tau$, tends to diverge below $15 \mathrm{~K}$ (see inset of figure 6). This behaviour is typical of what has been observed in many glass-formers in the $T$-regime of glass formation (Debenedetti and Stillinger 2001). The non-
Arrhenius behaviour of $\tau(T)$ resembles that of fragileglass former like o-terphenyl (Chattopadhyay et al 2005). The signature of the kinetic arrest of the FM-AFM transition is also visible in the isothermal $\mathrm{H}$-dependent measurements (Manekar et al 2001). This is in the form of a striking feature of the ZFC virgin $M-H$ curve lying out of the envelope. $M-H$ curve obtained by subsequent field cycling between $\pm H_{\max }$, where $H_{\max }>H_{\mathrm{M}}$. A very similar behaviour has now been observed in CMR Mn-oxides (Dho and Hur 2003) and magnetocaloric materials (Chattopadhyay et al 2004; Tang et al 2004).

\section{Interdisciplinary research gains}

Such S\&T activity in these technologically important functional magnetic materials is helping in the deeper understanding of the FOPT process. Study of a FOPT process involving the water-ice transition has remained a very active area of research since it has tremendous implications for the biological as well as ecological systems (Heneghan et al 2002). Although many aspects of a FOPT, viz. phase-coexistence and metastability (supercooling/ superheating), nucleation and growth are actually known intuitively to the metallurgists and crystal grower for the ages, a systematic and universal understanding of a FOPT process is still lacking. This is now more than an academic interest especially when it is becoming more and more apparent that a first order transition process is involved in many of the practical issues in our surroundings (Heneghan et al 2002). Some of these magnetic materials of current interest can actually be used as test bed materials to study a FOPT process in a two-parameter magnetic field $(H)-$ temperature $(T)$ phase space. Such exploration in conventional pressure $(P)$-temperature $(T)$ phase space is relatively difficult. Knowledge gathered in exploring $H-T$ phase space in magnetic materials can also be applied in other areas of technological interest viz. vortex matter phases of type-II superconductors and ferroelectric materials. While implications in ferroelectric materials are well known, the understanding of vortex matter phase space is absolutely necessary for tuning the dissipationless current carrying capacity of a type-II superconductor.

\section{Future outlook}

While GMR using multilayer structure is already a matured technology, CMR materials in spite of their initial promise are yet to reach that stage. However, the recent identification of the phase-coexistence as a crucial element to understand the CMR properties (Dagotto et al 2002) has raised the prospect of tuning their properties for technological applications. The progress from basic research to technological applications in magnetocaloric materials and magnetic shape memory alloys is also taking place very rapidly. It is clear that MCE is one of the most critical parameters 


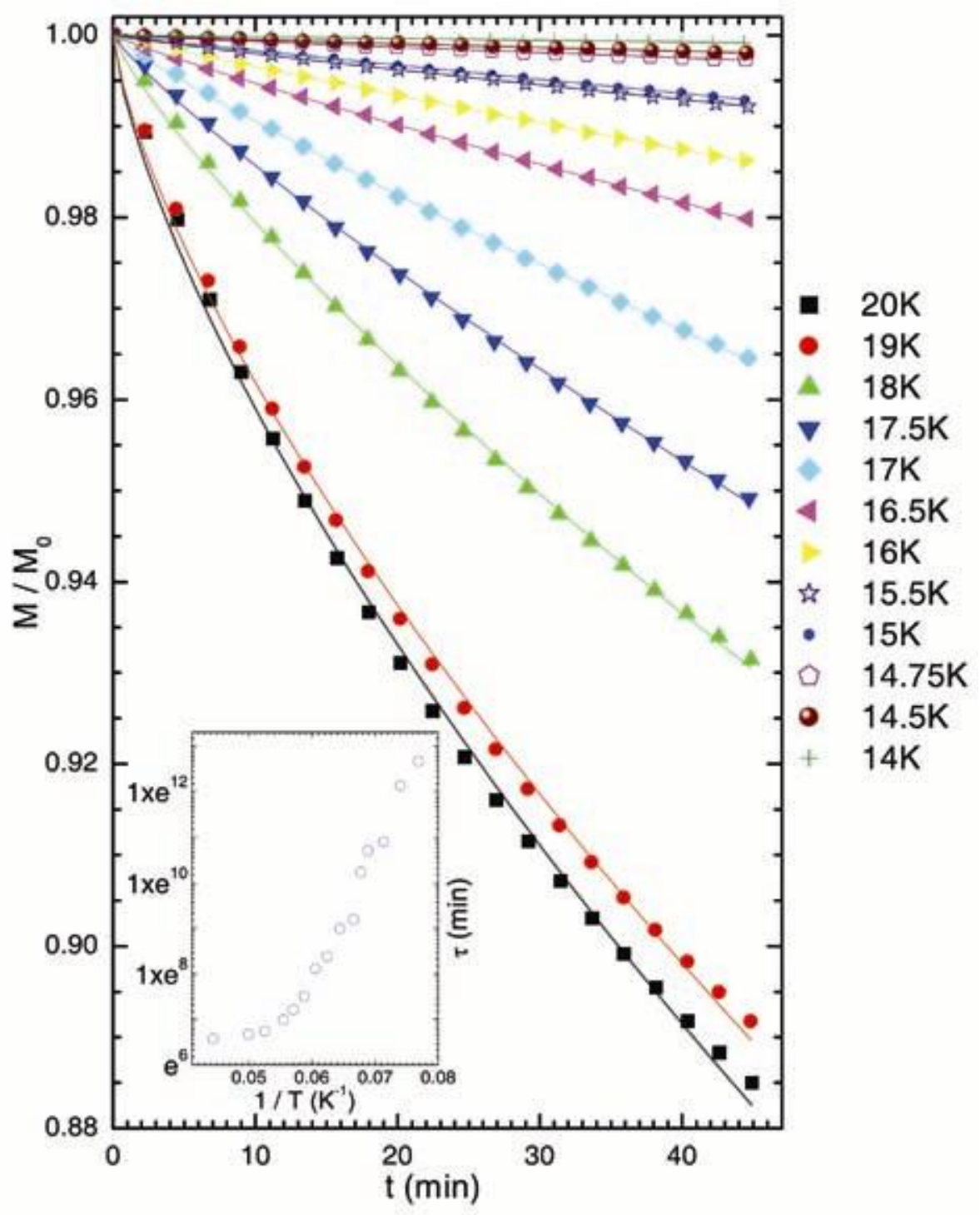

Figure 6. Normalized magnetization $(M)$ vs time $(t)$ plots for $\mathrm{Ce}\left(\mathrm{Fe}_{0.96} \mathrm{Ru}_{0.04}\right)_{2}$ sample at various fixed temperatures on the FCC path. The solid lines represent the fitting with Kohlrausch-Williams-Watt (KWW) exponential function. The inset shows the characteristic relaxation time, $\tau$ (obtained from the fitting of KWW function) as a function of $T$ (Chattopadhyay et al 2005).

defining the performance of a magnetic refrigerator. The stronger the MCE the higher the efficiency of the device, hence the hunt is on to find better MCE materials. It is interesting to note here that some of the CMR manganites and magnetic shape memory alloys are now found to be showing large MCE around room temperature. With improvements in magnetic materials, magnet systems and understanding of the active magnetic regenerator cycle, there is promise that a mature magnetic refrigeration technology will soon produce thermodynamic efficiencies comparable to or higher than the best available gas compression devices. It provides an example of closely intertwined science and technology reinforcing each other on the way from laboratory based basic research to useful futuristic technology.

\section{References}

Ahn K H et al 2004 Nature 428401

Bhattacharya K 2003 Microstructure of martensite (UK: Oxford University Press)

Brawer S 1985 Relaxation in viscous liquids and glasses (Columbus, Ohio: The American Ceramic Society Inc.)

Chaddah P and Roy S B 1999 Phys. Rev. B60 11926

Chaikin P M and Lubensky T C 1995 Principles of condensed matter physics (Cambridge UK: Cambridge University Press)

Chattopadhyay M K et al 2003 Phys. Rev. B68 174404

Chattopadhyay M K et al 2004 Phys. Rev. B70 214421

Chattopadhyay M K et al 2005 Phys. Rev. B72 180401R

Dagotto E 2002 Nanoscale phase separation and colossal magneto-resistance (Berlin: Springer) 
Debenedetti P G and Stillinger F H 2001 Nature 410259

Dho J and Hur N H 2003 Phys. Rev. B67 214414

Imry Y and Wortis M 1979 Phys. Rev. B19 3580

Kennedy S J and Coles B R 1990 J. Phys.: Condens. Matter 2 1213

Kimura T et al 1999 Phys. Rev. Lett. 833940

Majumdar S 2005 Solid State Commun. 13685

Manekar M A et al 2000a J. Phys. Condens. Matter 12 L409

Manekar M A et al 2000b J. Phys. Condens. Matter 129645

Manekar M A et al 2001 Phys. Rev. B64 104416

Moore J A et al 2006 Appl. Phys. Lett. 88072501

Pecharsky V K and Gschneidner Jr. K A 2001 Adv. Mater. 13683

Pecharsky V K et al 2004 Phys. Rev. Lett. 91197204
Prinz G 1998 Science 2821660

Roy S B and Coles B R 1989 J. Phys.: Condens. Matter 1419 Roy S B and Coles B R 1990 Phys. Rev. B39 9360

Roy S B and Chaddah P 1997a Physica C279 70

Roy S B and Chaddah P 1997b J. Phys.: Condens. Matter 9 L625

Roy S B et al 2002 J. Phys. Condens. Matter 149779

Roy S B et al 2004 Phys. Rev. Lett. 92147203

Roy S B et al 2005 Phys. Rev. B71 174413

Soibel A et al 2000 Nature (London) 406283

Sokhey K J S et al 2003 J. Phys. D36 1366

Sokhey K J S et al 2004 Solid State Commun. 12919

Tang H et al 2004 Phys. Rev. B69 064410 